\title{
Application of DIGE and mass spectrometry in the study of type 2 Diabetes Mellitus (T2DM) mouse models
}

Book or Report Section

Accepted Version

Smith, C., Mills, D. and Cramer, R. (2012) Application of DIGE and mass spectrometry in the study of type 2 Diabetes Mellitus (T2DM) mouse models. In: Cramer, R. and Westermeier, R. (eds.) Difference Gel Electrophoresis (DIGE). Methods in molecular biology (854). Humana Press / Springer, New York, pp. 299-318. ISBN 9781617795725 doi:

https://doi.org/10.1007/978-1-61779-573-2_21 Available at https://centaur.reading.ac.uk/30234/

It is advisable to refer to the publisher's version if you intend to cite from the work. See Guidance on citing.

To link to this article DOI: http://dx.doi.org/10.1007/978-1-61779-573-2_21

Publisher: Humana Press / Springer

All outputs in CentAUR are protected by Intellectual Property Rights law, including copyright law. Copyright and IPR is retained by the creators or other copyright holders. Terms and conditions for use of this material are defined in the End User Agreement. 


\section{CentAUR}

Central Archive at the University of Reading

Reading's research outputs online 


\section{Application of DIGE and Mass Spectrometry in the Study of Type 2 Diabetes Mellitus (T2DM) Mouse Models}

Celia Smith ${ }^{1}$, Davinia Mills ${ }^{2}$ and Rainer Cramer ${ }^{1,2^{*}}$

${ }^{1}$ Department of Chemistry, University of Reading, Reading, UK

${ }^{2}$ The BioCentre, University of Reading, Reading, UK

*Address correspondence to:

Prof Rainer Cramer, The BioCentre and Department of Chemistry, University of Reading, Harborne Building, Whiteknights, PO Box 221, Reading, RG6 6AS, UK.

Tel.: +44-118-378-4550; FAX: +44-118-378-4551; e-mail: r.k.cramer@rdg.ac.uk 
Summary

Knowledge of the differences between the amounts and types of protein that are expressed in diseased compared to healthy subjects may give an understanding of the biological pathways that cause disease. This is the reasoning behind the presented protocol, which uses difference gel electrophoresis to discover up- or down-regulated proteins between mice of different genotypes, or of those fed on different diets, that may thus be prone to develop diabetes-like phenotypes.

Subsequent analysis of these proteins by tandem mass spectrometry typically facilitates their identification with a high degree of confidence.

\section{Keywords}

Difference gel electrophoresis (DIGE), Type 2 diabetes mellitus (T2DM), Mass spectrometry, Proteomics, Quantitation 


\section{Introduction}

Type-2 diabetes mellitus (T2DM) has reached epidemic proportions globally and the number of sufferers is expected to more than double in the period 2000-2030 to 366 million worldwide(1, 2). The protocol presented here combines difference gel electrophoresis (DIGE) separation and quantitation with mass spectrometric identification of the proteins that are differentially expressed in lean and obese mice when fed standard and high fat diets. It is hoped that the identification of these proteins will help to unravel the complex biological pathways involved in glycaemic control and obesity, which are closely related to T2DM.

Five biological replicates of each mouse genotype/diet were chosen to obtain some statistical validity. Frozen liver samples from these animals were solubilised and labeled with one of two fluorescent dyes (red, Cy3, and blue, Cy5). Equal amounts of each of the underivatised samples were pooled and labeled with a third fluorescent dye (yellow, Cy2). The samples were then mixed and subjected to two-dimensional gel electrophoresis (2DE) so that per gel there were two samples of different fluorescent labels, plus an aliquot of the labeled pooled internal standard. Subsequent fluorescence scanning of the gels at each of the dyes' excitation/emission wavelengths yields gel images that can be superimposed, and in this image spots of red and blue indicate the more predominant proteins (see Figure 1).

Use of the pooled internal standard across all of the gels allows the gel imaging software to normalize the response for each gel. Thus, comparisons can be made to identify the proteins that are reproducibly either up- or down-regulated between samples. Protein spots of interest from one of the DIGE gels or a preparative gel, which is run simultaneously without fluorescent dye but with an increased amount of protein, can then be picked, subjected to tryptic digestion and analysed by LC-MS/MS. Protein identification is then made by matching actual peptide and fragment masses with theoretical equivalents from a sequence database. 
INSERT FIGURE 1 HERE.

2. Materials

2.1. Equipment

The 2DE equipment employed was an Ettan DALTtwelve System from GE Healthcare (Little Chalfont, Buckinghamshire, UK) and most of the associated hardware, consumables and manuals/protocols were from the same supplier.

\subsubsection{Protein Extraction from Mouse Liver Tissue}

1. Pestle and mortar

2. Ice-bath

3. Spectrophotometer

\subsubsection{Protein Labeling}

1. Ice-bath

2. Glass syringe, $500 \mu \mathrm{L}$

\subsubsection{Rehydration of IPG Strips}

1. IPG strips (3-10NL, $24 \mathrm{~cm}$; GE Healthcare)

2. Rehydration tray and lid (GE Healthcare)

\subsubsection{Isoelectric Focusing (IEF)}

1. Ettan IPGphor II with electrodes (GE Healthcare)

2. IEF ceramic tray and wicks (GE Healthcare)

3. 2 pairs of tweezers

4. Spirit level

\subsubsection{Second Dimension Gel Electrophoresis}


1. Rehydration tray and lid (GE Healthcare)

2. 2 pairs of tweezers

3. Thin but blunt long-bladed spatula

4. Microwave oven

5. Ettan DALTtwelve separation unit (GE Healthcare)

6. Rehydration tray and lid (GE Healthcare)

\subsubsection{Gel Scanning and Image Analysis}

1. Typhoon 9400 plus blue laser module and control software (GE Healthcare)

2. Gel alignment guide (GE Healthcare)

3. ImageQuant v5 software (GE Healthcare)

4. Progenesis SameSpots (Non-Linear Dynamics, Newcastle upon Tyne, UK)

\subsubsection{Visualization and Picking of Protein Spots of Interest}

1. Staining trays

2. Light box

3. Manual spot picker (e.g., One Touch Spot Picker, available from the Gel Company) and compatible tips with $1.5 \mathrm{~mm}$-orifice

4. Fine nose tweezers

\subsubsection{In-gel Digestion and NanoLC-MS/MS Analysis}

1. Sample concentrator (SpeedVac; Thermo Fisher Scientific, Hemel Hempstead, UK)

2. Dionex Ultimate 3000 with NCS-3500RS pump. NanoLC system comprising vacuum degasser, nanocapillary pump, loading pump, temperature-controlled column compartment, autosampler and Chromeleon Express control software (Dionex, Amsterdam, Holland) 
3. LTQ-Orbitrap XL mass spectrometer with nanoelectrospray ( $\mathrm{EESI}$ ) source and Xcalibur control software (Thermo Fisher Scientific)

4. Acclaim PepMap $100 \mathrm{LC}$ column, $75 \mu \mathrm{m}$ ID $\times 15 \mathrm{~cm}, 3 \mu \mathrm{C}_{18}$ packing, and a precolumn cartridge (300 $\mu \mathrm{m}$ ID x 0.5cm) packed with Acclaim PepMap 100 (Dionex)

5. Proxeon ES508 stainless steel emitters (Thermo Fisher Scientific)

6. Proteome Discoverer 1.0 software (Thermo Fisher Scientific)

7. In-house Mascot search engine (Matrix Science, London, UK)

\subsection{Consumables}

All solvents should be of HPLC-grade or equivalent, unless stated otherwise.

\subsubsection{Protein Extraction from Mouse Liver Tissue}

1. Liquid nitrogen

2. Lysis buffer at $\mathrm{pH} 8.3$ (8 $\mathrm{M}$ urea, $2 \mathrm{M}$ thiourea, 4\% CHAPS (w/w), $10 \mathrm{mM}$ Tris, $0.5 \%$ NP40)

3. Protein Assay (Bio-Rad, Hemel Hempstead, UK)

4. Bovine serum albumin (BSA) standards in the range of $0-2 \mathrm{mg} / \mathrm{mL}$ in water

5. Disposable semi-microcuvettes

\subsubsection{Protein Labeling}

1. N,N-dimethylformamide, anhydrous (DMF) (see Note 1)

2. CyDye DIGE Fluor minimal labeling kit, $2 \mathrm{nmol}$ or $5 \mathrm{nmol}$ (GE Healthcare)

3. $10 \mathrm{mM}$ solution of L-lysine monohydrochloride

4. Lysis buffer at $\mathrm{pH} 8.3$ (8 $\mathrm{M}$ urea, $2 \mathrm{M}$ thiourea, 4\% CHAPS (w/w), $10 \mathrm{mM}$ Tris, $0.5 \%$ NP40)

\subsubsection{Rehydration of IPG Strips}


1. $1.3 \mathrm{M}$ dithiothreitol (DTT; Sigma-Aldrich, Gillingham, UK)

2. IPG buffer with the same $\mathrm{pH}$ interval as the IPG strips (3-10NL; GE Healthcare)

3. Destreak rehydration solution (GE Healthcare)

4. DryStrip cover fluid (GE Healthcare)

5. IPGphor strip holder cleaning solution (GE Healthcare)

\subsubsection{Isoelectric Focusing}

1. Lint-free wipes

2. DryStrip cover fluid (GE Healthcare)

3. IPGphor strip holder cleaning solution (GE Healthcare)

\subsubsection{Second Dimension Electrophoresis}

1. SDS-PAGE gels

2. DTT

3. Iodoacetamide (IA; GE Healthcare)

4. Equilibration buffer (aqueous solution containing $50 \mathrm{mM}$ Tris, $6 \mathrm{M}$ urea, 30\% glycerol, $2 \%$ sodium dodecyl sulphate)

5. Agarose (ReadyPrep Overlay Agarose; Bio-Rad)

6. Tris-glycine-SDS run buffer, 10 times concentrate (National Diagnostics Protogel, obtainable from Fisher Scientific, Loughborough, UK)

2.2.6. Gel Scanning and Image Analysis

1. Water for plate cleaning

\subsubsection{Visualization and Picking of Proteins of Interest}

1. Fixing solution ( $40 \%$ ethanol, $10 \%$ acetic acid)

2. Destaining solution ( $1 \%$ acetic acid) 
3. Ammonium sulfate

4. Brilliant Blue $\mathrm{G} 250$

5. Phosphoric acid

6. Methanol

7. Ethanol 


\section{Methods}

To exclude the possibility of sample contamination with non-mouse proteins or other contaminants, it is advisable to carry out as many of the laboratory procedures as possible in a designated clean room or area. If this is not possible, great care must be taken at every step to avoid contamination, especially from human keratin which tends to be ubiquitous.

\subsection{Protein Extraction from Mouse Liver Tissue}

This protocol assumes that mouse liver tissues are frozen at $-80^{\circ} \mathrm{C}$. It is critically important to keep the liver tissues frozen until the lysis buffer has been added to the ground samples, to prevent proteases degrading the proteins.

The fact that liver tissue samples contain high levels of protein gives the advantage that it is not necessary to undertake additional sample clean-up steps. By taking only a small quantity of the liver lysate for DIGE, levels of possible interfering compounds will be low.

1. Add the mouse liver tissue to the pre-cooled mortar containing liquid nitrogen. Grind the frozen tissue to a fine powder, topping up the liquid nitrogen as it evaporates so that the tissue does not thaw.

2. Add a small amount (ca. $15-20 \mathrm{mg}$ ) of the powdered tissue to an Eppendorf tube and immediately add $1 \mathrm{~mL}$ of lysis buffer. Vortex and then sonicate the Eppendorf tube until the powdered tissue is dissolved.

3. Centrifuge the tube at $6^{\circ} \mathrm{C}$ and $12,000 \mathrm{~g}$ for 10 minutes to pellet the cell debris. Pipette off the supernatant, aliquot and store (as required) at $-80^{\circ} \mathrm{C}$.

4. Estimate the concentration of protein in the supernatant using a modification of the Bio-Rad Protein Assay, which is based on the Bradford method (3), by diluting the colour reagent five times with water, then adding $20 \mu \mathrm{L}$ of each sample or standard to $1.6 \mathrm{~mL}$ of the diluted colour reagent in a semi-microcuvette (see

Note 2). 


\subsection{Protein Labeling}

As two samples can be run per gel, the minimum number of gels will be half of the total number of samples to be analysed. Thus, if there are 20 samples to be run, it will be necessary to run ten gels. It is useful to prepare a randomized gel plan in advance, showing which samples will be labeled with the Cy3 and the Cy5 dyes, respectively, and how these will be combined on the gels. Table 1 shows such a plan for four different combinations of genotypes and diets and five biological replicates for each combination. Each gel is typically loaded with a total of $300 \mu \mathrm{g}$ of protein: $100 \mu \mathrm{g}$ from each of the two samples and a further $100 \mu \mathrm{g}$ from the pooled internal standard, which is labeled with the Cy2 dye.

1. Normalize the sample protein levels to $3 \mathrm{mg} / \mathrm{mL}$ by the addition of lysis buffer as necessary. This step greatly simplifies the protein labeling procedure. Check that the $\mathrm{pH}$ of each sample is between 8 and 9 (see Note 3 ).

2. To prepare the internal standard for labeling, pool an equal volume (amount) of $20 \mu \mathrm{L}(60 \mu \mathrm{g})$ of each sample in a suitable tube to give a sufficient volume (amount) of internal standard for all gels. Ensure the tube contents are wellmixed (see Note 4).

3. To prepare the individual samples for labeling, transfer for each individual sample twice the volume used in the previous step $(40 \mu \mathrm{L})$ into an Eppendorf tube for labeling with either Cy3 or Cy5 dye (see Notes 4 and 5).

4. Keep the samples on ice until you are ready to label them.

5. Prepare the CyDyes by addition of an appropriate volume of anhydrous DMF to each tube of the CyDye kit (see Note 6). The dye concentration of the resultant solution should be $400 \mathrm{pmol} / \mu \mathrm{L}$.

6. Usually $100 \mu \mathrm{g}$ of protein is labeled with $800 \mathrm{pmol}$ of dye. In the example presented, the amount of protein actually taken was $120 \mu \mathrm{g}$, and so $2.4 \mu \mathrm{L}$ of the appropriate CyDye solution is added to $40 \mu \mathrm{L}$ of the sample. 
7. As the total volume of the pooled internal standard is $20 \times 20 \mu \mathrm{L}=400 \mu \mathrm{L}(1200$ $\mu \mathrm{g})$, this is then labeled by the addition of $24 \mu \mathrm{L}$ of Cy2 dye.

8. The tubes containing the labeled samples and internal standards are vortexed briefly, spun and kept on ice in the dark for $30 \mathrm{~min}$.

9. To stop the labeling reaction at least an equal volume of $10 \mathrm{mM}$ lysine solution compared to the CyDye solution volume of steps 6 and 7 should be added to each tube. However, for convenience a higher volume, i.e. $5.6 \mu \mathrm{L}$ and $56 \mu \mathrm{L}$ respectively, should be chosen to increase the total volume by $20 \%$ to $48 \mu \mathrm{L}$ and $480 \mu \mathrm{L}$, respectively. All sample solutions now provide a sufficient number of 40 $\mu \mathrm{L}$ aliquots, each containing $100 \mu \mathrm{g}$ of sample, for ten gels to be run with two individual samples and one internal standard.

10. The samples are mixed, spun and kept on ice in the dark for a further 10 min and can then be either frozen and stored at this stage, or further prepared for the rehydration of the IPG strips (see Subheading 3.3).

\subsection{Rehydration of IPG strips}

In this procedure the labeled samples are firstly mixed in accordance with the gel plan (Table 1) and then reduced with DTT. Modified Destreak solution is added to make a total volume that is compatible with the IPG strip size and method of rehydration. In this example, where the $24 \mathrm{~cm}$-IPG strips are rehydrated in the presence of the protein sample, a maximum volume of $450 \mu \mathrm{L}$ is used.

1. Pipette $40 \mu \mathrm{L}$ of the Cy5-labeled sample into a $0.5 \mathrm{~mL}$-Eppendorf tube. Add 40 $\mu \mathrm{L}$ of the Cy3-labeled sample and $40 \mu \mathrm{L}$ of the Cy2-labeled internal standard. Repeat for all samples as detailed in the gel plan.

2. Add $22 \mu \mathrm{L}$ of the $1.3 \mathrm{M}$-DTT solution to each of the sample tubes and mix. 
3. Modify the Destreak solution by the addition of the IPG buffer - added here at $2 \%$ (see Note 7) - to give a sufficient volume for all samples, i.e. for the ten gels prepared in this example more than $(450-22-(3 \times 40)) \mu \mathrm{L} \times 10=3.08 \mathrm{~mL}$ is needed.

4. Add $308 \mu \mathrm{L}$ of modified Destreak solution to each tube, mix the tube contents and stand on ice in the dark for $30 \mathrm{~min}$.

5. Level the clean and dry rehydration tray in a convenient position on the bench and pipette each sample into an empty lane and record which sample is in which lane.

6. Using tweezers carefully peel off the backing strip for each IPG strip, and place gel-side down on top of the sample. Ensure there are no air bubbles trapped between the sample and the gel.

7. If the strips are the correct way up it should be possible to read the serial numbers of the IPG strips. Record these on the Gel Plan (Table 1).

8. Cover each lane with approximately $2 \mathrm{~mL}$ of DryStrip cover fluid, then slide the lid into place.

9. Protect from light and allow the strips to rehydrate overnight (see Note 8).

INSERT TABLE 1 HERE.

\subsection{Isoelectric Focusing (IEF)}

This is the first dimension of the separation, where voltage is applied across the strips to separate the proteins according to their pl. The voltage is increased in either a stepwise or gradient fashion to firstly remove ionic material to the ends of the strips, and then to gradually move the proteins to their pl. The IEF program detailed in Table 2 was found to be suitable for use with mouse livers. However, for any new study the program must be optimised empirically as there are many factors that can influence the separation, such as protein loading and concentration of the IPG buffer. 
IEF is conveniently carried out overnight. However, focusing for too long can cause horizontal streaking in the gels. In the program detailed in Table 2 the sole purpose of the last stage (S6 in Table 2) is to keep the proteins focused at low voltage until it is convenient to remove them from the IPGphor. The program can be stopped at any stage during S6, and the strips removed promptly before they are processed further (see Note 8).

1. Level the IPGphor II and ensure that the ceramic tray is completely clean and dry (see Note 9).

2. Place the ceramic tray onto the IPGphor II and carefully add approximately $108 \mathrm{~mL}$ of DryStrip cover fluid, ensuring that the fluid is evenly distributed across the lanes.

3. Using 2 pairs of tweezers carefully remove the IPG strips from the rehydration tray and drain off any surplus cover fluid onto a lint-free wipe. Ensure that the strip is placed gel-side up - the serial nos. should not be readable.

4. Place each strip, gel-side up, into a lane of the ceramic tray so that the end of the strip marked with "+" is furthest away from you.

5. Dampen each wick with $150 \mu \mathrm{L}$ of deionized water and place them at both ends of each IPG strip, so that they slightly overlap the ends of the gel (see Note 10).

6. Place each electrode assembly on top of the wicks at either end of the IPG strips. The assembly should make electrical contact with the IPG strips through the wicks, and with the IPGphor.

7. Add more DryStrip cover fluid to the tray so that the strips are completely covered, close the lid and protect the strips from light.

8. Set the IPGphor to run at $20^{\circ} \mathrm{C}$ and $75 \mu \mathrm{A} /$ strip. The program detailed in Table $\mathbf{2}$ has been successfully used for mouse livers.

9. Start the focusing program (Table 2) and enter the number of IPG strips when prompted. 
10. When focusing is complete (see Note 11), stop the focusing program, remove the strips and drain the DryStrip cover fluid onto lint-free paper without delay.

11. If not proceeding to the second dimension immediately it is possible to freeze the strips at $-80^{\circ} \mathrm{C}$ (see Note 8 ).

INSERT TABLE 2 HERE.

\subsection{Second Dimension Electrophoresis}

Before the proteins can be separated according to their molecular weight, it is first necessary to break down the three-dimensional structure of the proteins and to saturate the strip with sodium dodecylsulfate (SDS). This is done by equilibrating the strips in a cocktail, which includes a pH buffer, urea, SDS and a dye for monitoring the solvent front (see 2.2.5). Any disulfide bonds are reduced with DTT and then alkylated with IA. These equilibration steps are conveniently carried out in the rehydration tray.

1. If the strips have been frozen, allow them to thaw to room temperature.

2. If using the rehydration tray for the equilibration steps, the total amount of equilibration buffer needed will be $2 *$ [no. of IPG strips] $* 3 \mathrm{~mL}$ as there are two equilibration stages. This is then split into two equal aliquots.

3. Solid DTT is added to the first aliquot at $0.5 \%(\mathrm{w} / \mathrm{v})$, and solid IA is added to the second aliquot at $4.5 \%(\mathrm{w} / \mathrm{v})$. Both aliquots are placed on the roller shaker to dissolve the added contents; the IA-containing aliquot must be protected from light.

4. Place the IPG strips gel-side up in the rehydration tray and cover each strip with at least $2 \mathrm{~mL}$ of the equilibration buffer containing DTT. Ensure all of the strips are completely covered with the buffer.

5. Cover the tray with the lid and place on the orbital shaker for $15 \mathrm{~min}$.

6. Remove the strips from the tray and immerse each one briefly in a measuring cylinder containing diluted run buffer (see $\mathbf{2 . 2 . 5}$ ), then drain onto lint-free paper. 
7. Clean out the rehydration tray and ensure it is completely clean and dry.

8. Place the IPG strips gel-side up in the rehydration tray and cover each strip with at least $2 \mathrm{~mL}$ of the equilibration buffer containing IA. Ensure all of the strips are completely covered with the buffer.

9. Repeat steps 5 and 6.

10. Add $750 \mathrm{~mL}$ of concentrated run buffer to the Ettan DALTtwelve separation unit and add deionised water to the " 7.5 Liter" mark. Close the lid and turn on the pump to ensure thorough mixing of the contents.

11. With the $2^{\text {nd }}$ dimension gel assembly in an upright orientation add $1 \mathrm{~mL}$ of overlay agarose to the top of the gel using a pasteur pipette (see Note 12).

12. Lay the $2^{\text {nd }}$ dimension gel assembly horizontally on the bench with the opening facing you and the smaller of the two plates on top.

13. Using tweezers place the IPG strip gel-side up on the inner face of the larger of the two glass plates, with the "+" end facing towards the left as you look at it.

14. Quickly stand the gel cassette upright with the smaller glass plate in front (the " + " end of the IPG strip should now be pointing to the right).

15. Carefully use the spatula to push the IPG strip down into the agarose between the two glass plates (see Note 13).

16. Add more agarose if needed to completely seal the IPG strip into place. Allow the agarose to cool and set.

17. Repeat this procedure for all of the other $2^{\text {nd }}$ dimension gels and IPG strips.

18. Introduce the gel cassettes into the separation unit (see Note 14).

19. Dilute a further $200 \mathrm{~mL}$ of the concentrated run buffer to $2 \mathrm{~L}$ with deionised water. Add this to the top of the unit, ensuring that the final liquid level is between the MIN and MAX fluid levels. 
20. The Ettan DALTtwelve can conveniently be programmed to run overnight. It is advisable to focus the proteins at a low constant power level (e.g. $0.5 \mathrm{~W}$ per gel) for the first two hours, and then increase the wattage per gel to effect the separation. Optimum conditions will be found empirically, but in the work described in this chapter, a value of $2 \mathrm{~W}$ per gel was used (see Note 15).

21. The dye front appears as a thin blue line travelling slowly down the gel. When the dye front is close to the bottom of the gel the Ettan DALTtwelve should be stopped manually, and the gels scanned without delay.

\subsection{Gel Scanning and Image Analysis}

Gels must be scanned as soon as possible after the electrophoretic separation. Any delay could lead to diffusion of the proteins away from their tightly focused positions, which would broaden the size of the spot and increase the chance of contamination with other proteins.

Dust fluoresces and scatters light, which can cause artefacts on images and interfere with the subsequent quantitation. To minimize the chance of this happening, gels should only be handled wearing gloves, and these should be rinsed regularly with deionised water. The scanner should be completely clean and dust-free.

Conditions for the fluorescent scanning of the gels are first optimized quickly at low resolution, then all of the gels are scanned under ideal conditions at high resolution. Before scanning, gels are kept in the separation unit maintained at the sub-ambient temperature used for electrophoresis.

1. Turn on the Typhoon scanner $30 \mathrm{~min}$ before you intend to use it.

2. Clean the platen and position the gel alignment guide in place. 
3. Two gels can be scanned at the same time. Gels are positioned with the top opening towards the left and the smaller of the two glass plates in contact with the platen. See the manufacturer's manual for details and typical scanner settings.

4. To optimise the conditions, scan one of the gels at low resolution, typically using a pixel size of $500 \mu \mathrm{m}$. Review this scan using ImageQuant software, and quantify the intensity as denoted by "Max. Val(pos)" (see Figure 2). "Max. Val(pos)" must be less than 100,000 to avoid saturation. Optimum value for this parameter is in the range $50,000-80,000$. However, the PMT settings for the individual channels must be optimised so that broadly similar intensities are obtained for each channel.

5. Once the settings have been optimised, scan each gel in turn at high resolution, typically using a pixel size of $100 \mu \mathrm{m}$.

6. Store scanned gels damp at $4^{\circ} \mathrm{C}$ in plastic bags until ready to proceed with the visualization and spot-picking, which should commence as soon as possible after image analysis in order to reduce further protein diffusion and contamination.

\section{INSERT FIGURE 2 HERE.}

There are several 2D image analysis software packages that can be used to analyse the images from DIGE experiments and identify proteins that are either up or down-regulated. We have found Progenesis SameSpots to be fairly straightforward to use, and as it has plenty of on-screen help only the major steps will be covered in this protocol.

7. Open the program and load the gel images for DIGE analysis. The software permits cropping, flipping and rotating of images, and carries out several checks on the quality of the images before proceeding further. Images are organised according to the gels they originated from.

8. Select a reference image. All of the other images will then be aligned to this image, so it is advisable to pick as reference an image that is representative and free from 
streaking and distortion. Mask any area that is to be excluded from the analysis, for instance the very bottom of the gel that includes the dye front.

9. Align the images to the reference image. Each internal standard image (Cy2) is matched to the Cy2 image of the reference gel; the Cy3 and Cy5 images are then aligned to their respective Cy2 images. This is by far the most important step in the workflow. In practice an automatic alignment can be carried out as a first step; this can then be reviewed using the transition and checkerboard panes and any necessary modification can be made to the vectors.

10. Filter out any of the spots that you do not want to be included in the analysis such as reference markers that are placed at the edge of the gels.

11. Group the individual images together into their respective classes (e.g., diet 1 and diet 2, genotype 1 and genotype 2, control and treated).

12. Review each of the detected spots. There are certain criteria to be met in deciding which gel spots truly reflect a change in expression levels between any two conditions (diet, genotype, etc.). Tag all of the spots where the ANOVA p-value is $<0.05$ and fold change is greater than an appropriate level, e.g. 2.

13. From the "Progenesis Stats" icon, additionally tag those spots where statistical power is greater than $80 \%$ (the generally accepted threshold level). It is thus possible to scrutinise and reduce the list of detected proteins and by selecting only those peaks that have, for example, $p<0.05$, fold change $>2$, and power $>0.8$.

14. Visually examine these significant spots on the gel image to ensure that they can realistically be picked - and are not due to artefacts such as streaking.

\subsection{Visualization and Picking of Proteins of Interest}

Once the protein spots of interest have been identified they can be excised robotically or stained, visualized and excised manually. It is possible to excise from either one of the gels used in the DIGE experiment, or from a preparative gel that has been prepared and run 
simultaneously but without the use of fluorescent dyes, and with a much higher protein loading.

In this example, protein spots of interest were manually excised from the analytical gels. The staining method used has high sensitivity similar to that of silver staining - yet it is compatible with mass spectrometry (4). It is advisable to pick the spots from a gel where the protein spots of interest have a high intensity - for this reason it may be necessary to stain more than one gel.

1. Clean and dry the exterior of the gel cassette and lay it onto lint-free paper with the smaller of the two glass plates in contact with the paper.

2. Prise off the top glass plate carefully to avoid damaging the gel. Remove the IPG strip and agarose, and place the gel assembly gel-side up in a clean staining tray.

3. Cover the gel with fixing solution (see 2.2 .7 ) and place foil over the staining tray. Fix the gel overnight on a shaker.

4. Pour off the fixative and rinse the gel three times with deionized water.

5. To prepare one litre of colloidal coomassie stain, dissolve $100 \mathrm{~g}$ of ammonium sulphate in $500 \mathrm{~mL}$ of deionised water on a stirrer, and add $1.2 \mathrm{~g}$ of G250 Coomassie blue and $118 \mathrm{~mL}$ of phosphoric acid. When it has all dissolved, make the solution up to $800 \mathrm{~mL}$ with deionised water. Just before use add $200 \mathrm{~mL}$ of methanol dropwise with stirring.

6. Cover the fixed and rinsed gel with stain and place on a shaker for $16-40 \mathrm{~h}$.

7. Pour off the stain and rinse the gel three times with deionised water.

8. Add $250 \mathrm{~mL}$ of destaining solution and place on the shaker for at least 2 hours.

9. Once the gels have destained, scan them again on the Typhoon so that the coomassie image can be matched with the DIGE images in SameSpots (see Note 16 and manufacturer's manual for details). 
10. Import the Coomassie image(s) into the SameSpots software by selecting "Add images" from the Image QC screen. The software automatically checks the quality of the image before allowing further manipulation.

11. Select the "Spot Picking" screen and from there opt for "Picking manually".

12. The software then presents an image of the gel with the proteins of interest clearly identified. This image can be printed and used as a visual aid in the process of manual spot picking.

13. Choose the gel for spot excision and shake it in distilled water for 2 hours to remove any residual destaining solution.

14. Pre-wash the spot-picker tips with ethanol to remove any potentially leachable material that could interfere with the LC-MS/MS analysis. Allow the tips to dry.

15. Dry the back of the glass to which the gel is bonded to improve the view of the gel spots. Place it gel-side up onto the light-box (see Note 17).

16. Drop pure water onto the spot that is about to be picked.

17. Carefully lower the tip of the spot picker onto the gel so that the tip encircles the spot of interest. Press down slightly to pierce the gel, and then gently rotate the tip by one quarter turn to break the surface tension between the gel and the plate.

18. Carefully transfer the gel spot to an appropriately coded Eppendorf tube, and freeze at $-80^{\circ} \mathrm{C}$ until needed.

19. Repeat steps 16-18 for all of the spots of interest. Blanks and spots of major known proteins can also be excised as controls for the subsequent workflow.

20. Rescan the gel after picking the spots, as a record of where the spots were excised from.

\subsection{In-Gel Digestion and NanoLC-MS/MS Analysis}

Methods for in-gel digestion are well-documented in the literature; the method used here is based on the method published by Bindschedler et al. (5). Briefly, gel spots are 
repeatedly washed in ammonium bicarbonate solution with increasing amounts of acetonitrile, to remove the coomassie stain. After drying the spots are reduced with DTT, and cysteines are then alkylated with IA. After further washing and lyophilisation the gel spots are digested overnight with trypsin. Peptides are then extracted into a mixture of acetonitrile and trifluoroacetic acid, and are dried again and stored at $-80^{\circ} \mathrm{C}$ until ready for nanoLC-MS/MS analysis.

Typical experimental conditions for the nanoLC-MS/MS analysis are detailed ubiquitously in the literature. See section 2.1.10 for details of the instrumentation used in this example and Table $\mathbf{3}$ for typical results obtained from the comparison of different mouse genotypes. Note that in one case in Table $\mathbf{3}$ more than one gel was chosen for the analysis of the same protein spot.

INSERT TABLE 3 HERE. 
4. Notes

1. It is important to use anhydrous DMF for dissolving the fluorescent dyes. The DMF bottle should be sealed with a septum (to minimise ingress of air). If it was opened more than 3 months ago, it should not be used.

2. Dilution of the mouse liver lysates in water will be needed to bring the sample absorbance within the range of the calibration standards. Generally a ten times dilution will often suffice. This step will also help to avoid any interference in the colorimetric technique from high levels of urea present in the lysate.

3. Optimum $\mathrm{pH}$ values for labeling the sample are between 8 and 9. No pH adjustment was found to be necessary when using the lysis buffer described in 2.2.1 for the presented example. However, the $\mathrm{pH}$ of each set of samples should be checked before proceeding.

4. The amount of protein of each sample that will be pooled is equal to no. of gels*100 $\mu \mathrm{g}$ protein/no. of samples, which in the presented example equals $50 \mu \mathrm{g}$ per sample. If the protein concentration has been normalised at $3 \mathrm{mg} / \mathrm{mL}$, this is then equivalent to a nominal value of $16.67 \mu \mathrm{L}$ of each individual sample to be labeled with Cy2. To avoid problems due to volumetric losses, in the presented example this nominal volume of $16.67 \mu \mathrm{L}$ is increased to $20 \mu \mathrm{L}(60 \mu \mathrm{g})$. Volumetric losses can easily occur due to factors such as sample frothing, sample adhesion to pipette tips and tubes, evaporation and the cumulative effect of very slight pipettor volumetric inaccuracies. However, it is essential that all of the gels contain the same level of internal standard. - Analogously, the volume (amount) of individual samples taken for Cy3 or Cy5 dye labeling is increased to $40 \mu \mathrm{L}(120$ $\mu \mathrm{g}$.

5. It is helpful to colour-code the tops of the tubes with marker pens which are the same colour as the dye which will be added later. (Cy3 is red, Cy5 is blue and Cy2 
is yellow). This helps to minimise the chance of costly mix-ups at the gel preparation stage.

6. For the $2 \mathrm{nmol}-\mathrm{CyDye}$ kit, add $5 \mu \mathrm{L}$ of anhydrous DMF, and for the $5 \mathrm{nmol}-\mathrm{CyDye}$ kit add $12.5 \mu \mathrm{L}$ of anhydrous DMF. In order to label ten gels as described, it will be necessary to have two of the $5 \mathrm{nmol}$ - and one of the $2 \mathrm{nmol}$-CyDye kits.

7. In some cases it is more appropriate to add the IPG buffer at $0.5 \%$ as higher concentrations can limit the maximum voltage attainable during focusing and increase the time needed for this step. It is added here at $2 \%$ to maximise the solubility of the proteins.

8. If it is not convenient to process the strips directly after the rehydration or the IEF stage, they can be frozen at $-80^{\circ} \mathrm{C}$. However, they cannot be stored once the focussed strips have been equilibrated at the stage described in $\mathbf{3 . 5}$.

9. The ceramic tray must be cleaned with a detergent at neutral $\mathrm{pH}$ (for example, the proprietary cleaning solution sold for this purpose - listed in 2.2.4). Other detergents could strip off the surface of the tray, which has been treated to minimise protein adsorption.

10. During rehydration the gels swell and take on the blue colour of the modified Destreak solution. It is thus easy to see the ends of the (previously colourless) gels.

11. The blue colouration from the bromophenol blue (present in the DeStreak solution) quickly clears during focusing but this does not indicate that focusing is complete.

12. If ReadyPrep Overlay Agarose is used it can conveniently be warmed up in the microwave oven. If many gels are being prepared it may be necessary to reheat the agarose to prevent it becoming too viscous too soon. 
13. It is important not to damage either gel or strip. The spatula should only touch the backing of the IPG strip, not the gel itself. The IPG strip is in the correct place when it is touching the top of the $2^{\text {nd }}$ dimension gel and there are no air bubbles, only agarose between the two gels.

14. The Ettan DALTtwelve will hold up to 12 gel cassettes. If there are fewer than 12 gels to be run, the vacant places must be filled with blank cassette inserts. Wet the outside of each gel cassette with the diluted run buffer in the separation unit, thus facilitating its correct placement in the Ettan DALTtwelve.

15. When programming the Ettan DALTtwelve ensure that the pump is turned on, and that a suitable temperature (for instance, $10^{\circ} \mathrm{C}$ ) is used. A temperature of less than $10^{\circ} \mathrm{C}$ should not be used.

16. It is possible to scan the colloidal Coomassie images using a normal flat-bed scanner. However, the process of matching the coomassie image to the DIGE image is simplified if the Typhoon scanner is used throughout.

17. The light box used for gel-picking should be placed in an ergonomically advantageous position, and the environment should be as clean and dust-free as possible. Any contamination that is introduced after electrophoretic separation is likely to interfere with the nanoLC-MS/MS analysis of the proteins. 


\section{$\underline{\text { Acknowledgements }}$}

This work was supported by the EU $6^{\text {th }}$ Framework Programme "System-wide analysis and modelling of protein modification". The authors would additionally like to thank colleagues at the University of Reading (The BioCentre, Department of Chemistry and ICMR) and former colleagues at University College London (Proteomics Unit) for their invaluable advice. 
$\underline{\text { Tables }}$

Table 1: $\quad$ Example gel plan showing the labeling scheme for two genotypes (Gen1 and Gen2), two diets (Diet1 and Diet2) and five biological replicates of each possible combination of genotype and diet.

\begin{tabular}{|c|c|c|c|}
\hline Gel No. & Cy2 & Cy5 & Cy3 \\
\hline $\mathbf{1}$ & Pool & Gen1/Diet1-1 & Gen1/Diet2-1 \\
\hline $\mathbf{2}$ & Pool & Gen1/Diet2-2 & Gen2/Diet2-1 \\
\hline $\mathbf{3}$ & Pool & Gen2/Diet1-1 & Gen1/Diet1-2 \\
\hline $\mathbf{4}$ & Pool & Gen2/Diet2-2 & Gen2/Diet1-2 \\
\hline $\mathbf{5}$ & Pool & Gen1/Diet1-3 & Gen2/Diet1-3 \\
\hline $\mathbf{6}$ & Pool & Gen2/Diet2-3 & Gen1/Diet2-3 \\
\hline $\mathbf{7}$ & Pool & Gen2/Diet2-4 & Gen1/Diet1-4 \\
\hline $\mathbf{8}$ & Pool & Gen1/Diet2-4 & Gen2/Diet1-4 \\
\hline $\mathbf{9}$ & Pool & Gen2/Diet1-5 & Gen2/Diet2-5 \\
\hline $\mathbf{1 0}$ & Pool & Gen1/Diet1-5 & Gen1/Diet2-5 \\
\hline
\end{tabular}


Table 2: $\quad$ IPGphor program used in the isoelectric focusing of mouse liver lysates.

\begin{tabular}{|c|l|l|l|}
\hline Stage & $\begin{array}{l}\text { Step or } \\
\text { Gradient }\end{array}$ & Voltage & $\begin{array}{l}\text { Duration or } \\
\text { kV-hours }\end{array}$ \\
\hline S1 & Step & $150 \mathrm{~V}$ & $2 \mathrm{~h}$ \\
\hline S2 & Step & $500 \mathrm{~V}$ & $2 \mathrm{~h}$ \\
\hline S3 & Gradient & $1000 \mathrm{~V}$ & $3 \mathrm{~h}$ \\
\hline S4 & Gradient & $8000 \mathrm{~V}$ & $5 \mathrm{~h}$ \\
\hline S5 & Step & $8000 \mathrm{~V}$ & $96000 \mathrm{kVh}$ \\
\hline S6 & Step & $500 \mathrm{~V}$ & $2000 \mathrm{kVh}$ \\
\hline
\end{tabular}


Table 3: $\quad$ Partial Mascot results list of significant protein identifications for the differentially expressed proteins in T2DM mouse models.

\begin{tabular}{|c|c|c|c|c|c|c|c|c|c|}
\hline Spot & prot_desc & prot_score & prot_mass & prot_matches & prot_cover & prot_pi & Protein Name (from UniProtKB) & Fold Change* & p-value* \\
\hline 359 & Q8VC12 & 241 & 75227 & 24 & 7.4 & 7.27 & Urocanate hydratase & 2.4 NZO down & 3.87E-06 \\
\hline \multirow{6}{*}{420} & P32020 & 605 & 59715 & 23 & 30.5 & 7.16 & Non-specific lipid-transfer protein & \multirow{6}{*}{2.9 NZO down } & \multirow{6}{*}{$2.16 \mathrm{E}-08$} \\
\hline & Q8CHT0 & 479 & 62228 & 11 & 17.1 & 8.58 & Delta-1-pyrroline-5-carboxylate dehydrogenase & & \\
\hline & P24270 & 467 & 60013 & 13 & 22.6 & 7.72 & Catalase & & \\
\hline & Q99L88 & 135 & 58444 & 4 & 9.1 & 8.51 & Beta-1-syntrophin & & \\
\hline & Q03265 & 114 & 59830 & 2 & 4 & 9.22 & ATP synthase subunit alpha, mitochondrial & & \\
\hline & P24549 & 89 & 55060 & 2 & 5.6 & 7.92 & Retinal dehydrogenase 1 & & \\
\hline \multirow{4}{*}{481} & P11679 & 1648 & 54531 & 194 & 54.3 & 5.7 & Keratin, type II cytoskeletal 8 & \multirow{4}{*}{$2 \mathrm{NZO}$ up } & \multirow{4}{*}{$4.51 \mathrm{E}-13$} \\
\hline & Q63836 & 925 & 53147 & 118 & 36.7 & 5.78 & Selenium-binding protein 2 & & \\
\hline & Q91XD4 & 347 & 59529 & 11 & 15 & 5.79 & Formimidoyltransferase-cyclodeaminase & & \\
\hline & Q61035 & 102 & 57893 & 3 & 5 & 5.68 & Histidyl-tRNA synthetase, cytoplasmic & & \\
\hline \multirow{4}{*}{$481 \mathrm{~W}$} & Q63836 & 344 & 53147 & 13 & 18.6 & 5.78 & Selenium-binding protein 2 & \multirow{4}{*}{$2 \mathrm{NZO}$ up } & \multirow{4}{*}{$4.51 \mathrm{E}-13$} \\
\hline & P30416 & 188 & 51939 & 3 & 9.2 & 5.54 & Peptidyl-prolyl cis-trans isomerase & & \\
\hline & P11679 & 148 & 54531 & 4 & 8.6 & 5.7 & Keratin, type II cytoskeletal 8 & & \\
\hline & Q91XD4 & 75 & 59529 & 3 & 4.6 & 5.79 & Formimidoyltransferase-cyclodeaminase & & \\
\hline \multirow{5}{*}{$481 \mathrm{E}$} & P11679 & 1118 & 54531 & 30 & 47.3 & 5.7 & Keratin, type II cytoskeletal 8 & \multirow{5}{*}{$2 \mathrm{NZO}$ up } & \multirow{5}{*}{$4.51 \mathrm{E}-13$} \\
\hline & Q63836 & 884 & 53147 & 32 & 45.1 & 5.78 & Selenium-binding protein 2 & & \\
\hline & Q91XD4 & 179 & 59529 & 3 & 6.5 & 5.79 & Formimidoyltransferase-cyclodeaminase & & \\
\hline & Q61035 & 114 & 57893 & 2 & 4.9 & 5.68 & Histidyl-tRNA synthetase, cytoplasmic & & \\
\hline & P07724 & 124 & 70700 & 2 & 5.75 & 4 & serum albumin & & \\
\hline \multirow{4}{*}{484} & Q63836 & 1667 & 53147 & 113 & 71.4 & 5.78 & Selenium-binding protein 2 & \multirow{4}{*}{$5.2 \mathrm{NZO}$ up } & \multirow{4}{*}{$1.11 \mathrm{E}-15$} \\
\hline & P17563 & 1290 & 53051 & 102 & 55.7 & 5.87 & Selenium-binding protein 1 & & \\
\hline & Q91XD4 & 657 & 59529 & 14 & 29.2 & 5.79 & Formimidoyltransferase-cyclodeaminase & & \\
\hline & P11679 & 236 & 54531 & 6 & 11.2 & 5.7 & Keratin, type II cytoskeletal 8 & & \\
\hline \multirow{2}{*}{651} & Q9Z0S1 & 541 & 33517 & 47 & 35.7 & 5.54 & $3^{\prime}\left(2^{\prime}\right), 5^{\prime}-$ bisphosphate nucleotidase 1 & \multirow{2}{*}{2.9 NZO down } & \multirow{2}{*}{$2.24 \mathrm{E}-08$} \\
\hline & P21278 & 95 & 42283 & 3 & 8.4 & 5.7 & Guanine nucleotide-binding protein subunit alpha-11 & & \\
\hline 654 & Q9Z0S1 & 489 & 33517 & 45 & 28.2 & 5.54 & 3'(2'),5'-bisphosphate nucleotidase 1 & 5.5 NZO up & $3.16 \mathrm{E}-10$ \\
\hline 864 & P16015 & 542 & 29633 & 33 & 51.2 & 6.89 & Carbonic anhydrase 3 & 2 NZO down & $1.82 \mathrm{E}-12$ \\
\hline 865 & P16015 & 241 & 29633 & 10 & 20 & 6.89 & Carbonic anhydrase 3 & 2 NZO down & $3.30 \mathrm{E}-10$ \\
\hline
\end{tabular}

All identifications have at least two unique peptide matches in Mascot. Human and associated mouse keratins are excluded. ${ }^{*}$ These values were obtained from Progenesis SameSpots; proteins are either significantly up- or down-regulated for the genotype New Zealand Obese (NZO) in comparison to the genotype Swiss Jim Lambert (SJL). 
Figure Captions

Figure 1: Fluorescent DIGE image highlighting the differential proteins expressed for the Swiss Jim Lambert genotype fed on either a standard (Cy3-labeled, pink) or a high fat (Cy5-labeled, blue) diet (Gel 4 of Table 1).

Figure 2: Optimization strategy for the PMT settings for each of the three dye channels in ImageQuant (Cy3 only is shown here). 


\section{$\underline{\text { References }}$}

1. Wild, S., Roglic, G., Green, A., Sicree, R., and King, H. (2004) Global prevalence of diabetes: estimates for the year 2000 and projections for 2030, Diabetes Care 27, 1047-1053.

2. WHO. (2009) Fact Sheet No. 312: Diabetes.

3. Bradford, M. M. (1976) A rapid and sensitive method for the quantitation of microgram quantities of protein utilizing the principle of protein-dye binding, Anal Biochem 72, 248-254.

4. Candiano, G., Bruschi, M., Musante, L., Santucci, L., Ghiggeri, G. M., Carnemolla, B., Orecchia, P., Zardi, L., and Righetti, P. G. (2004) Blue silver: a very sensitive colloidal Coomassie G-250 staining for proteome analysis, Electrophoresis 25, 1327-1333.

5. Bindschedler, L. V., Palmblad, M., and Cramer, R. (2008) Hydroponic isotope labelling of entire plants (HILEP) for quantitative plant proteomics; an oxidative stress case study, Phytochemistry 69, 1962-1972. 


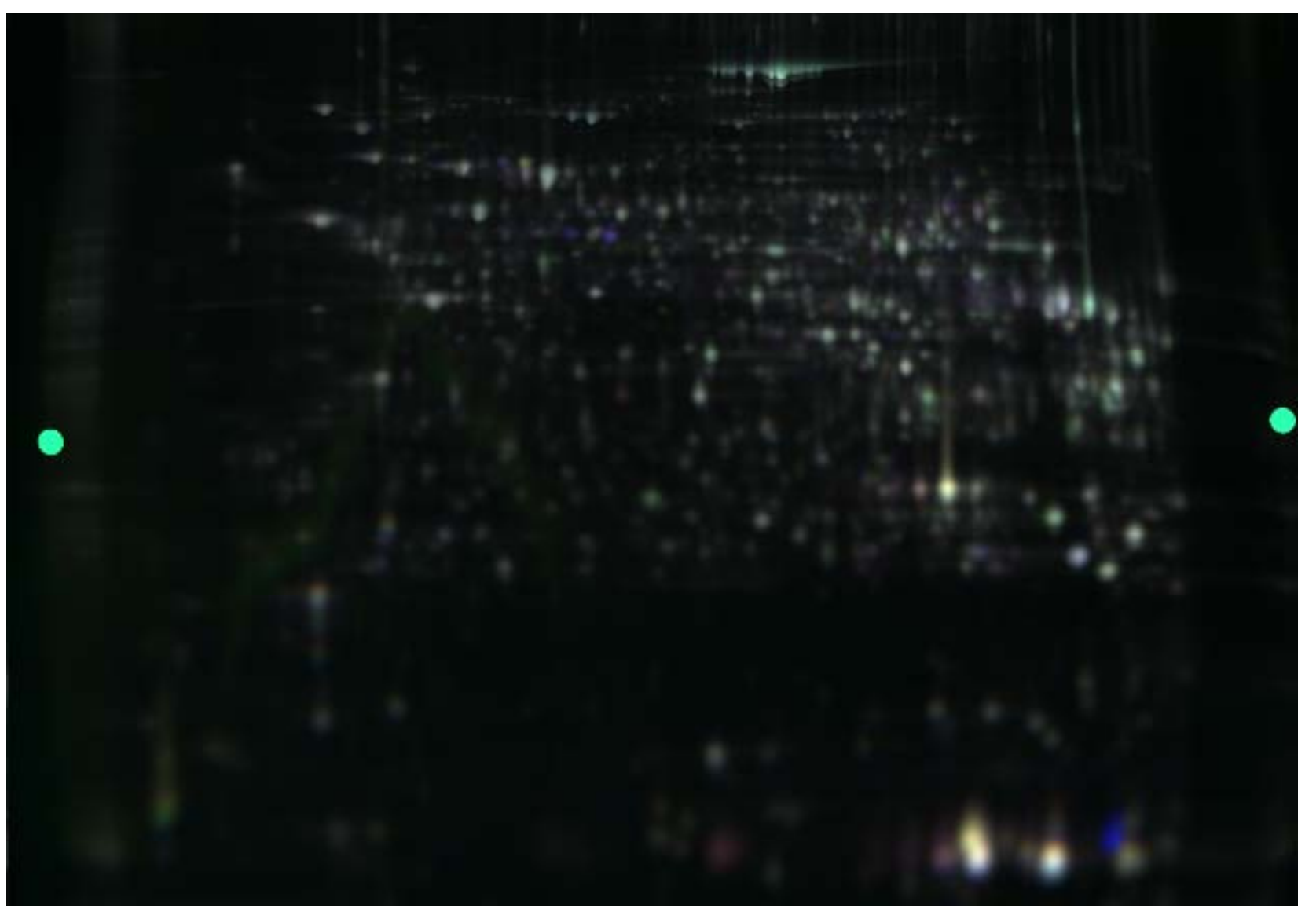

Figure 1 

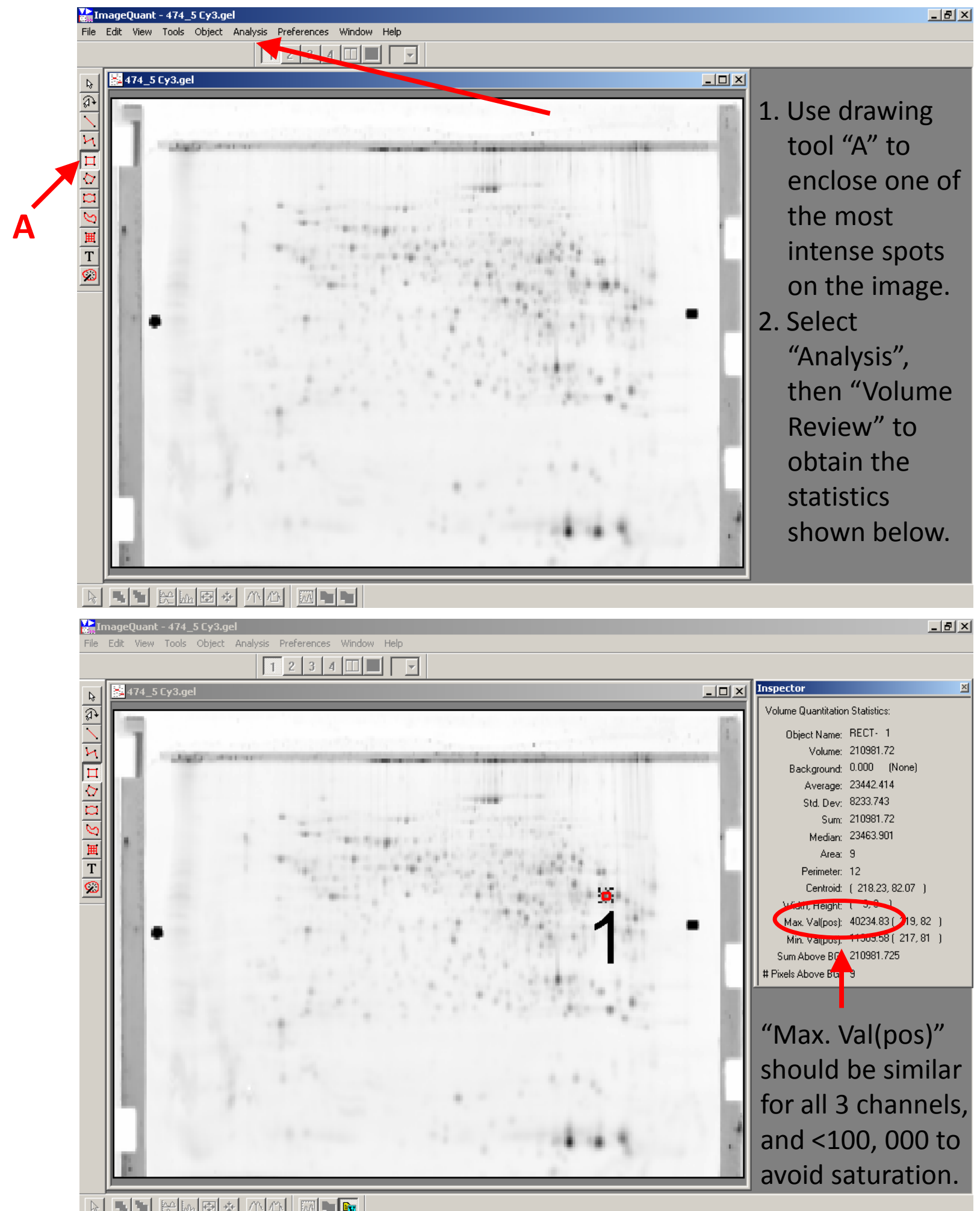

Figure 2 